\title{
Magneto-transport properties of dilute granular ferromagnets
}

\author{
A. Cohen A. Frydman and R. Berkovits ${ }^{1}$ \\ ${ }^{1}$ The Physics Department and the Minerva center, Bar-Ilan University, Ramat-Gan 52900, Israel
}

\begin{abstract}
We present magnetoresistance (MR) measurements performed on quench condensed granular $\mathrm{Ni}$ thin films which are on the verge of electric continuity. In these systems the electric conductivity is believed to be governed by the resistance between a very small number of grains. The films exihibit sharp resistance jumps as a function of magnetic field. We interpret these findings as being the result of magneto-mechanical distortions that occour in single grains which act as bottlenecks in the dilute percolation network. The observed features provide a unique measure of magnetostriction effects in nano-grain structures as well as being able to shed light on some of the properties of regular granular magnetic films.
\end{abstract}

Transport in granular metals, i.e. systems of metallic islands imbedded in an insulating matrix, has been an active area of research for a number of decades but many experimental findings are still far from being understood. Among these are the temperature dependence of the resistance $\left(R \alpha \exp \left[\frac{T_{0}}{T}\right]^{0.5}\right)$ and the large noise associated with granular films. The case in which the metallic islands are ferromagnetic is of particular interest since these systems show magnetoresistance (MR) properties which are reminiscent of the giant magnetoresistance phenomena characteristic of magnetic multilayers.

A natural way to treat granular metals is by considering a Miller-Abrahams resistor network in a similar manner to that of disordered systems [1]. Each pair of grains is represented by a resistor with conductance proportional to the tunneling probability between the grains [2]. In this representation the sample conductance is governed by "critical resistances" which act as bottlenecks for the conductance and dominate the electric transport of the percolation network. Since the density of these critical resistors is determined by the distribution of resistances one can expect that as the distribution becomes very large the distance between critical resistors (i.e. the correlation length of the electric percolation network) grows until eventually it should approach the sample size. In this limit the sample is critical and the behavior of any bottleneck grain will have large influence on the total conductance. In this paper we describe conductance measurements performed on granular Ni films in which the transport is believed to be governed by a very small number of grains. The samples exhibit unique sharp resistance jumps as a function of magnetic field. We attribute these jumps to buckling type magnetostriction deformation of bottleneck grains leading to abrupt changes in the sample conductivity.

An established method to fabricate systems of granular metals in general [3, 4, 5, 6] and granular ferromagnets in particular [7, 8, 9] in a very controlled way is quench condensation. In this method thin films are grown by sequential evaporation on a cryogenically cold substrate under UHV conditions while monitoring the film thickness and resistance. If the samples are quench condensed

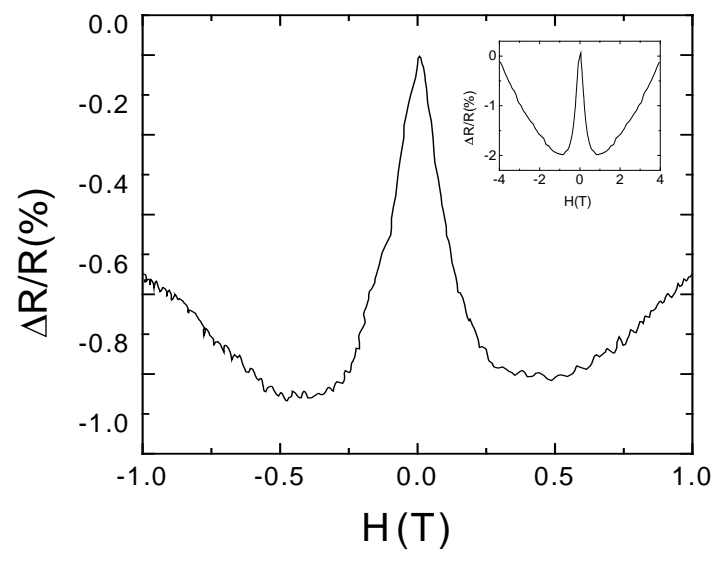

FIG. 1: Typical magnetoresistance for a conventional 2D quench condensed granular Ni film having resistance of $1 \mathrm{M} \Omega$ at $\mathrm{T}=4.2 \mathrm{~K}$. The insert shows a large field $\mathrm{MR}$ of a similar sample showing that the positive MR trend continues to very large fields.

on a non-passivated substrate such as $\mathrm{SiO}$, they begin growing in a granular manner so that the film is constructed of separated islands. As more material is quench condensed, the average distance between the islands decreases and the resistance drops. In these samples there is a critical thickness $d_{C}$, below which no conductivity can be practically measured. Once the thickness, $d$, of the sample is larger than $d_{C}$, the sheet resistance, $\mathrm{R}$, drops exponentially with $d$ reflecting the tunneling nature of the transport in these systems.

The quench condensation method provides a very sensitive control on the sample growth process, allowing one to terminate the evaporation at any desired stage of the material deposition and "freeze" the morphological configuration. In particular, it allows one to stop the film growth at a thickness for which a measurable conductivity first appears across the sample $\left(d \approx d_{C}\right)$. The current study was performed on quench condensed granular $\mathrm{Ni}$ films which were prepared under these conditions. Recently, we have studied a number of quench condensed granular materials in-which deposition was terminated 
at the verge of electric conductivity [10]. These show a number of features which differ from the usual 2D macroscopic systems and are characteristic of mesoscopic samples . Among these are the following [10]:

- Resistance switches and induced noise appear randomly (as a function of time) in many of these samples. There are also large sample to sample variations in the transport properties.

- Superconducting films show magnetoresistance oscillations similar to those which are observed in $1 \mathrm{D}$ superconducting granular wires [1]. The area associated with these oscillations is $(700 \AA)^{2}$.

- As in macroscopic granular films, these samples exhibit an electric field dependence of the form $\mathrm{I} \propto e^{\frac{F_{0}}{F^{\frac{1}{3}}}}$ for large electric field, F. However, the effective sample length extracted from the measured $\mathrm{I}-\mathrm{V}$ curves is three orders of magnitude smaller than those of macroscopic systems.

These findings lead us to consider the possibility that despite the fact that these samples have macroscopic dimensions $\left(\sim 0.5 \mathrm{~cm}^{2}\right)$, the percolation network is dilute enough so that the transport is governed by a very small area of the sample. This observation is supported by our measurements of the magneto-transport of granular ferromagnets described below. We name these samples "dilute samples" as opposed to the regular 2D films.

The magnetoresistance of 2D quench condensed Ni has been extensively studied in the past [7, 8, 9]. A typical MR trace is shown in figure 1 . The field in these experiments was applied perpendicular to the film. The MR curve shows a resistance peak centered at $\mathrm{H}=0$ followed by negative MR up to fields of $\pm 0.5 T$. This behavior is similar to that observed in insulating granular ferromagnets prepared by co-evaporation of a magnetic material and an insulation material [12, 13, 14, 15, 16] and is a result of spin dependent tunneling between grains which have randomly oriented magnetic moments 17. Applying a magnetic field aligns these moments causing a resistance decrease. The magnitude of the MR in our films is always of the order of $1-2 \%$ at $\mathrm{T}=4 \mathrm{~K}$. A unique feature that appears in all of the high-resistance quench condensed films is the increase of the resistance at high fields $(\mathrm{H}>0.5 \mathrm{~T})$ rather than a saturation that is expected for large $\mathrm{H}$ where all the grain moments are assumed to be aligned. This finding has been a puzzling issue for several years. As we note below, the results presented in this current work may shed some light on this high-field positive MR trend.

The MR curves of the dilute samples show several unique features that are not seen in the regular granular systems. These are depicted in figures 2 and 3 where it is seen that at low fields the samples exhibit a negative MR similar to those of regular 2D films. However, at
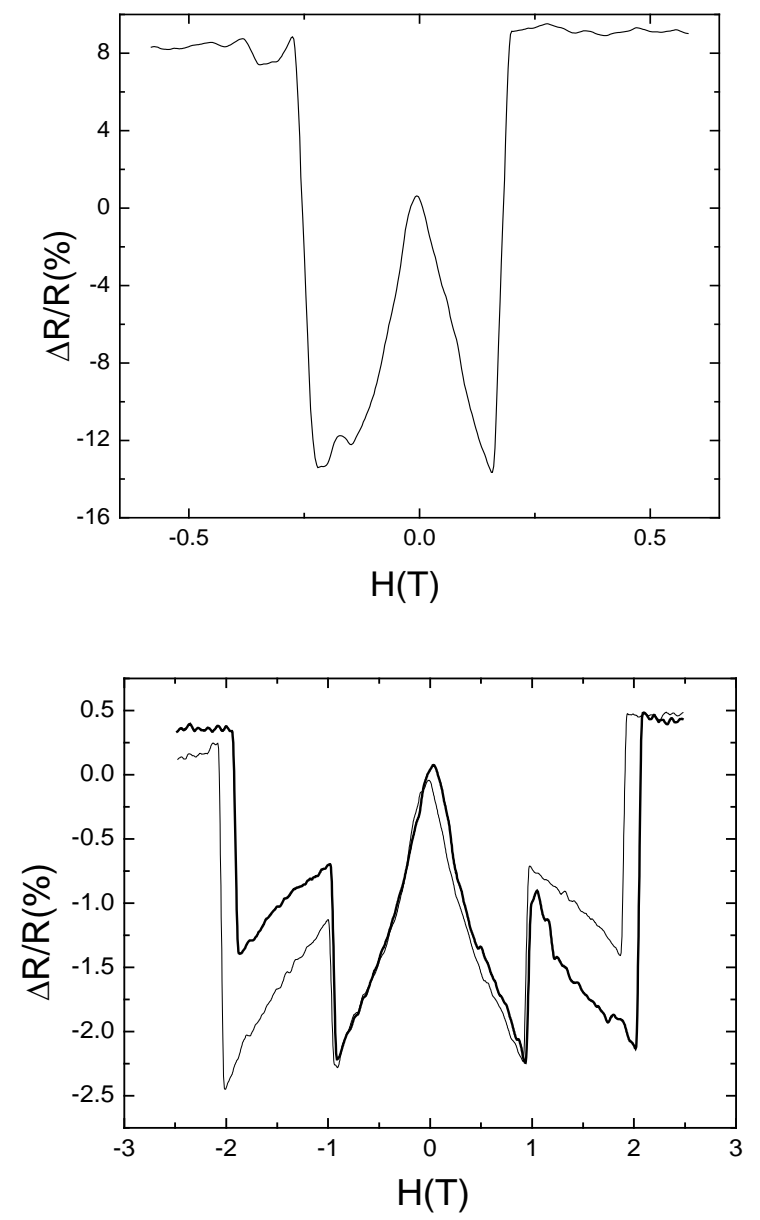

FIG. 2: Magnetoresistance traces of dilute granular Ni samples in which the quench condensation was terminated close to the threshold for electric continuity. The top frame is an example for a sample that exhibits a single jump. The bottom frame shows the MR of a sample showing a double-jump structure. The heavy line is for a field swept from left to right and the light line is for the opposite sweep

specific magnetic fields the resistance switches abruptly to a higher value. In some cases we observe a single resistance jump, such as that seen in the top frame of figure 2, after which the resistance saturates at a high resistance value. In other cases we observe two jumps (figures 2 bottom frame and figure 3 ). The first is a relatively small resistance increase followed by the continuation of the negative MR trend and the second is a large resistance jump leading to saturation at a high value. It is worth noting that although the qualitative behavior at low fields is similar to that of usual $2 \mathrm{D}$ granular systems, the magnitude of the negative MR in our samples can be much larger. While the MR reported in previous works on granular ferromagnets rarely acceded $2 \%$, we commonly observe effects of the order of $10 \%$ and in some cases the MR is as large as $60 \%$ (see figure 3). Clearly, in these films there is a large sample-to-sample variation in the magnitude of the negative $M R$ and of 


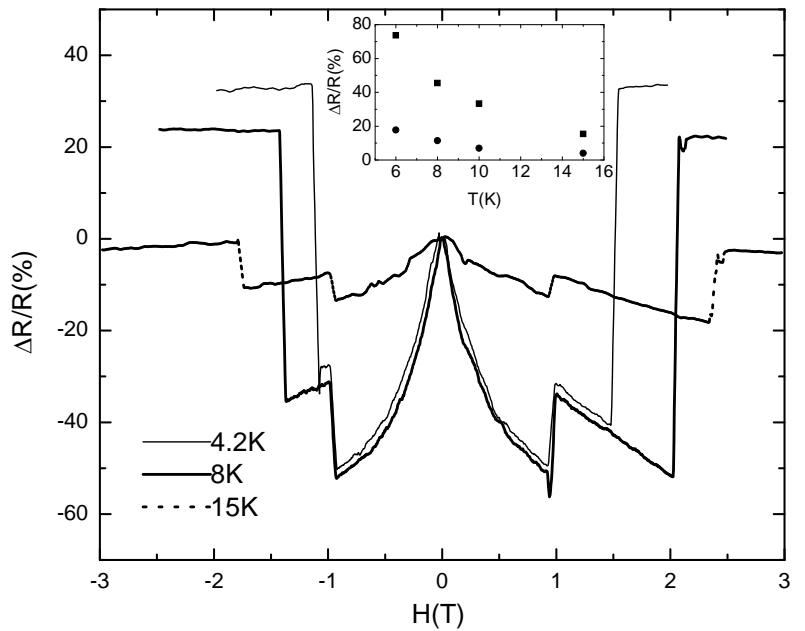

FIG. 3: The temperature dependence of a MR curve. The insert shows the dependence of the amplitude of the two resistance jumps (circles for the first jump and squares for the second) on temperature. The amplitude extrapolates to zero at around $\mathrm{T}=35 \mathrm{~K}$.

the sharp jumps as well as the in the magnetic field at which the jump occurs. This feature further reflects the mesoscopic nature of these geometries.

A number of properties of the MR curves should be noticed. First, although the traces are very reproducible, the jumps are hysteretic as seen in figure 2. Sweeping the field in opposite directions produces mirror images of MR. Second, raising the temperature results in two clear trends (figure 3): The magnitude of the jumps decreases with raising the temperature in a similar manner to that of the negative MR at low fields, and, at the same time the magnetic field of the jumps shifts to higher values. Finally, perhaps the most surprising finding is the fact that the saturated resistance value at high magnetic fields is larger than that of the value at $\mathrm{H}=0$. At zero field the magnetic grain moments are believed to be totally random and the resistance is expected to be maximal. The high resistance value at high magnetic fields is therefore a very unexpected result.

In an attempt to understand the above behavior we observe that the electric properties of these samples may be dominated by a small number grains which are characterized by the largest inter-grain distance that participate in the conduction paths. We have considered the possibility that the jumps are a result of magnetic moment flip of such a bottleneck grain. Recently, Meilikhov [18] considered the behavior of small single-domain grains in an external magnetic field and showed that their magnetic moment may flip abruptly if the initial angle between the magnetic field and the easy axis of the grain is larger than $\pi / 2$.If a bottleneck grain is subject to such magnetic moment flip the MR of the entire sample conductance would be subject to sharp features.

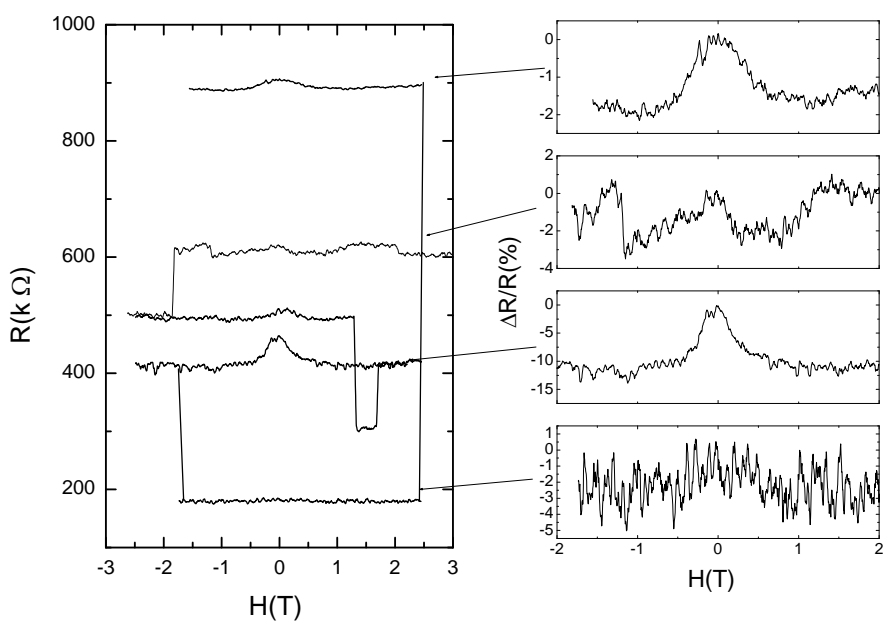

FIG. 4: Resistance versus magnetic field of a dilute sample showing pronounced switching behavior while sweeping the magnetic field back and forth many times. The right frames are the zoom on the MR curves of the different branches

However, the above model seems to be inconsistence with a number of our observations. In the first place this scenario could naturally explain sharp resistance decreases. Our samples always exhibit sharp increases in the resistance. Furthermore, the resistance value after the jump is higher than that at $\mathrm{H}=0$ where the grain moment orientations are assumed to be completely random. Secondly, a pre-requirement for a resistance jump is that the bottleneck grain moment orientation have a larger-than- $\pi / 2$-angle with the magnetic field direction. Our grains are believed to be in the superparamagnetic state, and it is hard to see how this condition is fulfilled. But even if such a situation occurs, computer simulations on such a granular configuration show that if the grain moment begins with such an angle, the initial MR would be positive rather than negative. In addition to the above, according to the Meilikhov picture the field at which the jump occurs should decrease with increasing temperature since the thermal energy assists in overcoming the anisotropy energy. This is opposite to the trend observed in figure 3 .

We have also considered the possibility that the resistance jumps are associated with switches between different current trajectories leading to sudden resistance changes. Such a process also doesn't seem to explain our observations because of the reproducibility of the data. The jumps are always upwards, they are always of the order of magnitude of the negative MR itself and the curves trace each-other when sweeping the field many times. Random switches between different trajectories are expected to cause either increase or decrease of the resistance with the same probability, to be random in size and magnetic field and to be non-reproducible. Indeed, a number of our samples showed much larger resis- 
tance switches which were random both in field and in sign. An example is shown in figure 4. It is seen that after each switch the magnetoresistance curve follows a different trace having different MR magnitudes (ranging between $0.5-13 \%$ ). In particular, one of the traces shows a resistance jump similar to the effects described above. It seams reasonable to assume that this sample (unlike that of figures 1,2 and 3 ) is characterized by a number of bottleneck trajectories and the current switches randomly between these paths leading to the different MR fingerprints.

The above considerations lead us to suggest that the effects seen in our samples are due to magneto-mechanical deformation of the grains. Magnetostriction (MS) in bulk $\mathrm{Ni}$ is well known and widely studied but not a lot is known about such effects in nano-structures. Geometry changes in small grains are extremely difficult to measure and, in granular films which are achieved by coevaporation, the grains are imbedded in an insulating matrix and any MS effects depend strongly on the elasticity of this insulator. The quench condensed systems provide us with a unique opportunity to study MS effects on small grains since they are free standing particles. We envision that as the grains start aligning parallel to each other, all perpendicular to the substrate, and dipole-dipole interactions cause magnetic repulsion between the grains. At some magnetic field, a buckling instability may occur and a small MS distortion of the grains will take place causing a conductance decrease. Since the conductance is achieved by tunneling, any small distortion (even by fractions of an $\AA$ ) in the geometry of the grains that belong to a conduction bottleneck will have a large effect on the conductivity. We estimate the magnetostatic interactions between two neigbouring parallelly aligned grains to be approximately $70 \mathrm{~K}$ (about an order of magnitude larger than the thermal energy of the system at the relevant measurements). Our experience shows that increasing the temperature of quench condensed grains above $40 \mathrm{~K}$ causes annealing and irreversible morphology changes in the sample. It seems reasonable, therefore, that magnetic forces in our samples could also have a considerable effect on the morphology of the grains. According to this model, if an external field aligning the grains is applied perpendicular to the substrate, two opposing effect influence the resistance. One is the fact that the tunneling probability is higher for aligned grains. This acts to lower the resistance causing a negative MR background. The second is a magnetostatic repulsion causing the intergrain distance to increase slightly, thus decreasing the tunneling probability at the buckling instability fields.

The model described above can shed light on some of the features observed in the MR of usual 2D quench condensed granular samples. As noted above, one of the puzzling features in these samples is the increase of the MR at high magnetic fields at which the resistance is expected to have reached saturation (see figure 1). This anomaly is observed mainly when the resistance of the granular films is relatively high. Such behavior can be explained by magnetic repulsion between grains causing minute geometrical changes which decrease the inter-grain tunneling probabilities causing resistance increases. In the dilute systems, this manifests itself as sharp resistance jumps while in regular samples one observes a continuous mild increase in resistance with increasing field. Such an effect can be particularly expected in quench condensed samples in which the grains are free standing on a substrate separated by vacuum. In granular samples, which are achieved by co-evaporation of metal and insulating materials, geometrical modifications are less probable.

In summary, we have fabricated and measured dilute granular Ni films in which the transport is believed to be governed by a very small number of grains. These samples exhibit sharp resistance increases at discrete magnetic fields. In order to explain these findings we proposed a qualitative model which involves buckling of bottleneck grains due to magnetostatic repulsion. The unique geometry of our samples enables one to detect the properties of a single grain even if the sample has macroscopic dimensions. Our granular samples also provide a unique opportunity to study magneto-mechanical effects on nanometer sized magnetic structures. The results obtained on these extreme samples shed some light on the behavior of the ferromagnetic granular samples in general and they may also be relevant for the development of new types of magneto-machanically based single-grain devices.

We gratefully acknowledge illuminating discussions with R.C. Dynes, Z. Ovadyahu and M. Pollak. This research was supported by the Binational USA-Israel fund grant number and by the Israeli academy of science.

[1] ambegaokar V. Ambegeokar, B.I. Halperin and .S. Langer, Phys. Rev. B4, 2162 (1971).

[2] C.J. Adkins, J. Phys. C: Solid State Phys., 15, 7143 (1982).

[3] M. Strongin, R. Thompson, O. Kammerer and J. Crow, Phys. Rev. B1, 1078 (1970).

[4] R.C. Dynes, J.P. Garno and J.M. Rowell, Phys. Rev Lett. 40, 479 (1978)

[5] H.M. Jaeger, D.B. Haviland, B.G. Orr, and A.M. Goldman, Phys. Rev B40, 182 (1989).

[6] R.P. Barber and R.E. Glover III, Phys. Rev. B42, 6754 (1990).

[7] A. Frydman and R.C. Dynes, Solid state commun.

[8] A. Frydman, T.K. Kirk and R.C. Dynes, Solid State Commun.

[9] A. Frydman and R.C. Dynes, Phil. Mag.

[10] A. Frydman et al in preparation.

[11] A.V. Herzog et at, Phys. Rev. B58, 14199 (1998)

[12] J.I. Gittelman, Y. Goldstein and S. Bozowski, Phys. Rev. B5, 3609 (1972).

[13] A. Milner, A. Gerber, B. Groisman, M. Karpovsky and 
A. Gladkikh, Phys. Rev. Lett. 76, 475 (1996).

[14] W. Yang, Z.S. Jiang, W.N. Wang and Y.W. Du, Solid State Commun. 104, 479 (1997).

[15] S. Honda, T. Okada, M. Nawate and M. Tokumoto, Phys. Rev. B56 14566 (1997).

[16] S. Sankar and A.E. Berkowitz, App. Phys. Lett. 73535
(1998).

[17] J.S. Helman and B. Abeles, Phys. Rev. Lett. 37, 1429 (1976).

[18] E.Z. Meilikhov, JETP, 891184 (1999). 